\title{
Darf der Staat das ethisch Richtige anordnen? Zur Arbeit der Forschungsethikkommissionen
}

\author{
Markus Zimmermann-Acklin
}

Online publiziert: 22. Januar 2010

(C) Springer-Verlag 2010

Es gehört inzwischen zum internationalen Standard, Forschungsvorhaben, die Menschen mit einbeziehen, einer Forschungsethikkommission zur Beurteilung vorzulegen. Ähnlich wie in Deutschland sind die damit befassten Ethikkommissionen auch in der Schweiz dezentral organisiert und Teil eines staatlichen Aufsichtssystems, das sowohl die Qualität der Forschung als auch den Schutz der involvierten Personen sicherstellen soll. Um das zu ermöglichen, haben die interdisziplinär zusammengesetzten Ethikkommissionen in der Regel die Einhaltung wissenschaftlicher, rechtlicher und ethischer Standards zu beurteilen und auf dieser Grundlage über die Zulassung klinischer Studien rechtswirksam zu entscheiden [6]. Die Aufgabe, rechtskräftige Entscheidungen treffen zu müssen, unterscheidet die Tätigkeit von Forschungsethikkommissionen grundlegend von derjenigen anderer Ethikkommissionen, welche beispielsweise für die Bereiche der klinischen oder politischen Ethikberatung zuständig sind und dort ausschließlich beratende Funktion ausüben.

Über die Frage, was die Forschungsethikkommissionen genau zu prüfen haben, ist in der Schweiz ein interessanter Konflikt entbrannt. Im Rahmen der Schaffung eines Bundesgesetzes über die Forschung am Menschen, einem neuen Humanforschungsgesetz, stand zur Debatte, ob die Forschungsethikkommissionen ausschließlich die Einhaltung rechtlicher oder darüber hinaus auch ethischer Normen zu überprüfen hätten. Entsprechend war auch die Erwähnung der „Ethik“ in der Bezeichnung der Kommissionen umstritten, insofern aus Sicht einiger Juristinnen und Juristen zutreffender von Prüfungs- oder Kontrollkommissionen die Rede sein sollte. Zu einer ersten Gesetzesfassung von 2006 hieß es in den Erläuterungen der Bundesverwaltung: „Kriterien wie ,voraussichtliche Akzeptanz des Forschungsprojekts in der Öffentlichkeit' oder, ethische Vertretbarkeit', die in der heutigen Beurteilungspraxis teilweise zur Anwendung kommen, dürfen zukünftig nicht mehr berücksichtigt werden. Dahinter steht der Grundsatz, dass der Rechtsstaat ausserhalb klarer rechtlicher Kriterien nicht das ,ethisch oder moralisch Richtige‘ anordnen und daran rechtsverbindliche Wirkungen anknüpfen darf. Eine Einschränkung der Forschungsfreiheit (...)

M. Zimmermann-Acklin $(\bowtie)$

Institut für Sozialethik, Universität Luzern, Gibraltarstrasse 3, 6000 Luzern 7, Schweiz

E-Mail: markus.zimmermann@unilu.ch 
ist nur auf der Basis gesetzlich normierter Kriterien zulässig (...)“([2], S. 20). Entsprechend sollten im Erstentwurf für das Humanforschungsgesetz von 2006 die Ethikkommissionen gemäß Zweckartikel lediglich die Erfüllung rechtlicher Anforderungen überprüfen; außerdem sollten sie die Durchführung von Forschungsprojekten und den Betrieb von Biobanken beaufsichtigen.

In der öffentlichen Vernehmlassung des Gesetzes ist dieser Vorschlag allerdings auf Ablehnung gestoßen. Im entsprechend angepassten Gesetzesentwurf [1], der in dieser Fassung demnächst im Parlament diskutiert werden wird, heißt es neu im Zweckartikel der Ethikkommissionen, sie hätten im Rahmen ihrer Zuständigkeiten zu überprüfen, ob die Studien und deren Durchführung den ethischen, rechtlichen und wissenschaftlichen Anforderungen des Gesetzes entsprächen. Insbesondere hätten sie zu prüfen, ob der Schutz der betroffenen Personen gewährleistet sei. In einem zusätzlichen Absatz wird hervorgehoben, sie könnten auch Forscherinnen und Forscher insbesondere zu ethischen Fragen beraten und auf Anfrage Stellungnahmen zu Forschungsvorhaben abgeben, die nicht dem Gesetz unterstehen, womit insbesondere Forschungsprojekte aus dem sozialwissenschaftlichen Bereich gemeint sein dürften, welche im Humanforschungsgesetz nicht einbezogen sind. Nicht mehr die Rede ist von der Beaufsichtigung von Forschungsprojekten und Biobanken, mit welcher die ehrenamtlich tätigen Kommissionen offensichtlich überfordert gewesen wären.

Da die Kommissionen rechtlich verbindliche Entscheidungen zu treffen haben, ist die Klärung der von ihnen zu prüfenden Normen von großer Bedeutung sowohl für die Forscherinnen und Forscher als auch für die Glaubwürdigkeit des Rechtssystems. Beide Vorschläge stellen Kompromisse dar: Würden die Kommissionen in Zukunft als Kontrollkommissionen bezeichnet, die ausschließlich die Einhaltung des Rechts zu prüfen hätten, würde die explizite Berücksichtigung ethischer Normen und damit eine bisher bewährte Praxis aufgegeben. Werden sie, wie im gegenwärtigen Gesetzesvorschlag der Fall, weiterhin Ethikkommissionen genannt, die u. a. die Einhaltung ethischer Kriterien zu überprüfen haben, bleibt eine gewisse Rechtsunsicherheit bestehen, insofern sich rechtlich nicht eindeutig bestimmen lässt, was in einem ethischen Überprüfungsverfahren genau und in welchem Maße gewichtet werden soll. Was hier geschieht, ist - neben einer spannenden Debatte über das Verhältnis von Ethik und Recht - eine Folge des Verrechtlichungsprozesses von Ethik: Da Ethikkommissionen nicht nur beraten, sondern rechtlich verbindliche Entscheidungen zu fällen haben, erhalten sie gesundheitspolizeiliche Aufgaben, können daher auch haftungsrechtlich belangt werden und es werden rechtliche Rekurse gegen ihre Entscheide möglich. Ethische Gremien begeben sich gleichsam freiwillig in ein Korsett rechtlich vorgegebener Normen und Prozeduren, um in heiklen Entscheidungsprozessen präsent bleiben zu können.

Aus rechtstaatlicher Sicht könnte derselbe Prozess auch als eine Ethisierung des Rechts beschrieben werden: Ethische Normen werden aus der moralischen in die rechtliche Sphäre übernommen und erhalten dadurch eine stärkere Verbindlichkeit, als sie zuvor im so genannten Soft-Law, also in internationalen und nationalen Ethikrichtlinien hatten. Diese Ethisierung des Rechts stößt dort an eine Grenze, wo Ermessensspielräume zu groß werden bzw. rechtlich verankerte Normen zu unbestimmt bleiben. Ethische Kriterien könnten dann zu einer Art Blackbox werden, wie es im oben stehenden Zitat zum Ausdruck gebracht wird, wenn es heißt, der Rechtsstaat dürfe nicht das ethisch oder moralisch Richtige anordnen.

Beispiele für im Gesetzesvorschlag bestehende Ermessensspielräume bieten die Interpretation der Angemessenheit oder Zumutbarkeit von Risiken und Belastungen, die Bestimmung minimaler Risiken und Belastungen insbesondere bei der Forschung mit Urteilsunfähigen, oder auch die neu geforderte Relevanz der Forschung für Wissenschaft und öffentliche Gesundheit. Würden die Auslegungspraxen regionaler Ethikkommissionen zu heterogen und Entscheidungskulturen zu unterschiedlich, führte dies wohl zu Recht zu Unmut bei For- 
schenden und Sponsoren. Um dies zu verhindern, haben sich die Ethikkommissionen in der Schweiz zu einer Arbeitsgemeinschaft zusammengeschlossen, welche sich um die Koordination und Vereinheitlichung der Vorgehensweisen, die Vertretung nach Außen und die Ausund Weiterbildung der Kommissionsmitglieder bemüht (http://www.swissethics.ch).

Insofern ethische Normen wie die zu achtende Menschenwürde, die Sicherung der Forschungsfreiheit und des Persönlichkeitsschutzes, die informierte Zustimmung, ein vertretbares Nutzen-Risiko-Verhältnis, die Vermeidung von Interessenskonflikten, die öffentliche Relevanz von Studien etc. [3] ins Recht aufgenommen wurden, umfasst die Prüfung rechtlicher Normen eo ipso auch die Prüfung ethischer Normen. Angesichts der möglichen Gefährdung besonders vulnerabler Gruppen ist es daher zu begrüßen, dass die Überprüfung der Studien durch eine von Forschung und Politik möglichst unabhängige Ethikkommission geschieht. Ein besonders heikler Bereich ist dabei die Forschung an Urteilsunfähigen, beispielsweise Kindern. Der Entwurf des Humanforschungsgesetzes sieht vor, dass Studien an Kleinkindern auch ohne direkten erwartbaren Nutzen durchgeführt werden dürfen, wenn diese nur mit minimalen Risiken und Belastungen verbunden sind und Erkenntnisse erwarten lassen, die für Personen mit derselben Krankheit längerfristig einen Nutzen ermöglichen könnten. Damit möchte der Gesetzgeber sicherstellen, dass auch die lange vernachlässigte Forschung an Kindern ermöglicht und die mit diesem Verzicht verbundenen Nachteile aufgehoben werden können.

An diesem Punkt gehen die Meinungen allerdings auseinander. Wichtige Argumente betreffen die durch die fremdnützige Forschung möglicherweise missachtete Menschenwürde der Kinder [7, 9], die Voraussetzung einer so genannten Minimalsolidarität und deren Begründung [5, 8], die schwierige Präzisierung dessen, was unter minimalen Risiken und Belastungen verstanden werden soll [9], die fragliche Unterscheidung von selbst- und eigennütziger Forschung in der klinischen Praxis bzw. den prinzipiellen Ausschluss vulnerabler Gruppen aus der Forschung [4]. Grundsätzlich wird die fremdnützige Forschung an Urteilsunfähigen unter Einhaltung gewisser Bedingungen im Bereich des Soft-Law auch in der Helsinki-Deklaration, im Bereich des Rechts in der von der Schweiz ratifizierten Biomedizin-Konvention des Europarats zugelassen.

Der Staat sollte nicht das moralisch Richtige anordnen. Durch die Einrichtung von Forschungsethikkommissionen sollte er hingegen ermöglichen, dass erstens die Studien und deren Durchführung den wissenschaftlichen, ethischen und rechtlichen Standards genügen und dass zweitens vulnerable Gruppen - neben Kindern und Jugendlichen sind auch Schwangere, Menschen mit Demenzerkrankungen oder mit Behinderungen betroffen - geschützt, jedoch nicht vollständig aus der Forschung und dem damit verbundenen Fortschritt ausgeschlossen werden.

\section{Literatur}

1. Entwurf eines Bundesgesetzes über die Forschung am Menschen (2009) http://www.bag.admin.ch/themen/medizin/00701/00702/07558/index.html?lang=de\#. Zugegriffen 11. Dez. 2009

2. Eidgenössisches Departement des Innern (2006) Bundesgesetz über die Forschung am Menschen (Humanforschungsgesetz, HFG). Erläuternder Bericht zum Vorentwurf. Bern

3. Emanuel EJ, Wendler D, Grady C (2000) What makes clinical research ethical? JAMA 283:2701-2711

4. Kind C (2007) „Fremdnützige“ Forschung mit Kindern - ist die scharfe Abgrenzung zu ,therapeutischer" Forschung adäquat und zweckmässig? Bioethica Forum 53:2-5

5. Maio G (2006) Das Kind als Forschungsobjekt? Ethische Überlegungen zur fremdnützigen Forschung mit Kindern. NZZ 185:67

6. SAMW (2009) Forschung mit Menschen. Ein Leitfaden für die Praxis. Basel 
7. Schwander V, Rütsche B (2008) Verletzt Forschung mit Kindern deren Würde? Zum Entwurf eines Verfassungsartikels über die Forschung am Menschen. NZZ 75:17

8. Seelmann K (2008) Wieweit reicht die Minimalsolidarität? Schwierige Abgrenzungen bei Forschung an Urteilsunfähigen. NZZ 24:19

9. Seelmann K, Kipfer D (2006) Der Zweck heiligt auch bei der Forschung nicht die Mittel. Rechtliche Grenzen wissenschaftlicher Forschung an Kindern. NZZ 185:65 